\title{
Accelerated convergence for Schrödinger equations with non-smooth potentials
}

\author{
Emil Kieri*
}

April 3, 2013

\begin{abstract}
When numerically solving the time-dependent Schrödinger equation for the electrons in an atom or molecule, the Coulomb singularity poses a challenge. The solution will have limited regularity, and high-order spatial discretisations, which are much favoured in the chemical physics community, are not performing to their full potential. By exploiting knowledge about the jumps in the derivatives of the solution we construct a correction, and show how this improves the convergence rate of Fourier collocation from second to fourth order. This allows for a substantial reduction in the number of grid points. The new method is applied to the higher harmonic generation from atomic hydrogen.
\end{abstract}

Keywords. Time-dependent Schrödinger equation, spectral methods, non-smooth coefficients, higher harmonic generation.

AMS subject classification. 35Q40, 65M12, 65M70.

\section{Introduction}

We consider higher harmonic generation (HHG) from an atomic gas exposed to a laser beam [17]. The electrons are bound in orbit around the nucleus, trapped by a Coulomb potential. The electric field of the laser beam causes the potential to tilt. A potential barrier is then formed through which one of the valence electrons may tunnel, ionising the atom. Since the electric field is oscillatory the tilt perpetually changes directions, accelerating detached electrons back and forth. If an electron recombines with the ion, returning to a bound orbit, a high-energy photon is emitted. The frequency of the emitted photon is, in semiclassical approximation, an odd overtone of the laser frequency, and is called a harmonic. The process is sketched in Figure 1. The HHG process has extensive applications in experimental physics as the generator of short, coherent pulses covering the spectral range from ultraviolet to x-ray [10]. Such pulses can be used e.g. for time-resolved spectroscopy of electron dynamics $[1,2]$. For different applications, different properties of the harmonic radiation are desirable. One often wants as high intensity as possible for a certain, application dependent,

\footnotetext{
*Department of Information Technology, Uppsala University, Box 337, SE-751 05 Uppsala, Sweden (emil.kieri@it.uu.se).
} 
frequency. The spectrum of generated harmonics is dependent of the properties of the incident laser pulse. By shaping the pulse it is possible to tailor the harmonic spectrum. HHG is a highly non-linear process, finding the desired pulse shape is therefore non-trivial. Much work has gone into optimising the harmonic spectrum with respect to a certain target experimentally by varying the incident laser pulse, see e.g. the review [17]. The aim of this paper is to improve the accuracy of simulation of the HHG process, as well as other applications involving electron dynamics. An application we have in mind is computational optimisation of the harmonic spectrum, for which efficient simulation of the process is a necessary component. The method developed is however not restricted to HHG, but applicable to any electron dynamics problem in cylindrical coordinates.

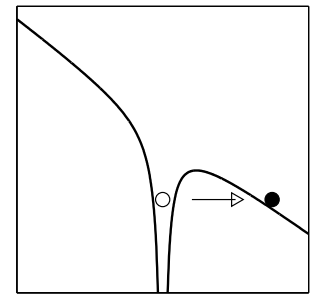

(a)

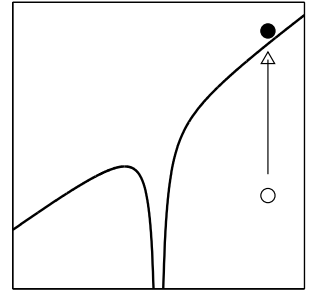

(b)



(c)

Figure 1: Semi-classical sketch of the HHG process. (a) The electric field of the laser beam tilts the potential. The electron may then tunnel through the potential barrier and ionise the atom. (b) The potential tilts in the other direction. The electron is then accelerated back towards the nucleus. (c) The electron recombines with the nucleus. A high-energy photon is emitted in the process.

Our model is the time-dependent Schrödinger equation (TDSE) for an electron in an atomic potential subject to a linearly polarised electric field. For the remainder of this paper we will consider the TDSE of atomic hydrogen due to its simplicity, but the method presented is applicable to any atom. In a multi-electron system one would use a simplified "single active electron" model considering only the highest occupied state, which will dominate the HHG. The other electrons would be modelled by an effective potential. The resulting model will have the same computational challenges as the one for the hydrogen atom. Posed in cylindrical coordinates the problem reads

$$
\begin{aligned}
\mathrm{i} u_{t} & =H u=-\frac{1}{2} \Delta u-\frac{1}{\sqrt{r^{2}+z^{2}}} u-z e(t) u, \quad r, t \geq 0,-\infty<z<\infty, \\
u(r, z, 0) & =u_{0}=\sqrt{2} \mathrm{e}^{-\sqrt{r^{2}+z^{2}}} .
\end{aligned}
$$

The problem has no angular dependence, the model is therefore two-dimensional. The coordinate system is centred at the nucleus with the $z$-axis aligned with the electric field. We neglect the force due to the magnetic field, which is small at the laser intensities considered. The wave function $u$ contains all retrievable information about the electron. The squared modulus of the wave function is a probability density, the probability of finding the electron in the subset $\Omega$ of the domain is $\int_{\Omega}|u|^{2} r \mathrm{~d} r \mathrm{~d} z$. The initial wave function $u_{0}$ is taken as the atomic 
ground state, i.e. the first eigenstate of $(1)$ with $e(t) \equiv 0$, and is normalised in the $L_{2}$ inner product

$$
(u, v)=\int_{r=0}^{r=\infty} \int_{z=-\infty}^{z=\infty} u^{*}(r, z) v(r, z) r \mathrm{~d} r \mathrm{~d} z .
$$

The normalisation is preserved during propagation since the Hamiltonian $H$ is self-adjoint with respect to this inner product. Except where explicitly stated otherwise atomic units are used throughout this paper. The elementary charge, the reduced Planck's constant $\hbar$, Coulomb's constant $\frac{1}{4 \pi \varepsilon_{0}}$ and the electron rest mass then all adopt the value one.

The Coulomb potential $\frac{-1}{\sqrt{r^{2}+z^{2}}}$ is singular at the origin. This poses a challenge for numerical methods, especially if high order of accuracy is desired, since it results in low regularity of the solution. When solving the TDSE numerically high order methods are often used. The free Schrödinger equation, $\mathrm{i} u_{t}=-u_{x x}$, with a single frequency initial condition $\mathrm{e}^{\mathrm{i} \omega x}$ is analogous to the advection equation $u_{t}+\omega u_{x}=0$. The growth with frequency of the phase velocity makes the Schrödinger equation more sensitive to dispersion errors compared to hyperbolic equations. This makes high order methods favourable. Due to its exact representation of dispersion relations, the Fourier pseudospectral method has been and is very popular in the chemical physics community [9]. The low regularity of the solution to (1), however, impairs the accuracy of the Fourier method. The goal of this paper is to recover some of this lost accuracy. Trigonometric interpolation of discontinuous functions suffers from the Gibbs phenomenon, spurious oscillations which do not decay as the number of basis functions is increased. If the function is continuous but non-smooth the interpolant is convergent, but at a rate limited by the smoothness of the function. Much work has gone into the study of impacts of and remedy to the Gibbs phenomenon. A thorough review is given by Tadmor in [15]. Fornberg studied numerically the impact of discontinuous coefficients for the Fourier method applied to the elastic wave equation [3, 4], and Luo and Kreiss considered the accuracy of finite difference and Fourier methods for the advection equation with discontinuous coefficients by analysing eigenexpansions [12]. In [8], Jung solves the advection equation with a Dirac delta distribution as source term using Chebyshev collocation. By approximating the delta distribution by the Chebyshev approximation of the derivative of a step function, Gibbs oscillations from the source term cancel the Gibbs oscillations due to the discontinuous solution and spectral accuracy is recovered once the initial oscillations have left the domain. In our problem, the lack of smoothness lies not in a source term, but in a coefficient. By adding a correction term in the same spirit, we are able to cancel the leading order error terms, increasing the order of accuracy.

\section{Radial discretisation and time integration}

In this section we will discretise the radial direction of the problem using Bessel functions. This softens the Coulomb singularity such that the potential becomes bounded and continuous. We will then introduce the temporal discretisation, the Magnus midpoint rule, and show that it preserves regularity properties of the solution which will be exploited when we construct the axial discretisation. 
We truncate the domain to the cylinder $r \in\left[0, L_{r}\right]$. The radial direction is discretised using the basis functions [16, Ch. 18]

$$
v_{k}(r)=\frac{\sqrt{2}}{L_{r} J_{1}\left(x_{k}\right)} J_{0}\left(\frac{x_{k} r}{L_{r}}\right), \quad k=1, \ldots, N_{r} .
$$

$J_{n}$ is the $n$ :th order Bessel function of the first kind, and $x_{k}$ is the $k$ :th zero of $J_{0}$. Note that $v_{k}\left(L_{r}\right)=0 \forall k$, i.e. we have a homogeneous Dirichlet boundary condition at $r=L_{r}$. This is an open boundary problem, we need a big enough computational domain for the solution to stay in its interior in order to get reliable results.

The functions $v_{k}$ have two favourable properties. They are orthonormal with respect to the weight $r$, which appears in the volume element for cylindrical coordinates, and they are eigenfunctions to the radial part of the Laplacian. We expand the solution in $\left\{v_{k}\right\}$ with coefficients dependent of the axial coordinate $z$ and time,

$$
u=\sum_{k=1}^{N_{r}} \varphi_{k}(z, t) v_{k}(r)
$$

and construct a Galerkin spectral method over the radial coordinate by multiplying (1) by a basis function $v_{k}$ and integrating $\int \cdot r \mathrm{~d} r$. This yields

$$
\mathrm{i} \dot{\varphi}_{k}(z, t)=\frac{1}{2}\left(\frac{x_{k}}{L_{r}}\right)^{2} \varphi_{k}(z, t)-\frac{1}{2} \frac{\partial^{2}}{\partial z^{2}} \varphi_{k}(z, t)+\sum_{j=1}^{N_{r}} W_{k j} \varphi_{j}(z, t)-z e(t) \varphi_{k} .
$$

Here, some of the terms end up diagonal due to the orthonormality of the functions $v_{k}$. In particular, we get an identity mass matrix. The equations are coupled through the potential matrix

$$
W_{j k}(z)=-\int_{0}^{L_{r}} v_{j}(r) v_{k}(r) \frac{r}{\sqrt{r^{2}+z^{2}}} \mathrm{~d} r, \quad j, k=1, \ldots, N_{r}
$$

which can be pre-computed. We use the notation $V(z, t)=W(z)-z e(t) I_{N_{r}}$ for the full, time-dependent potential. $I_{N_{r}}$ denotes the $N_{r} \times N_{r}$ identity matrix. On matrix-vector form, the system of partial differential equations for the $\varphi_{k}$ 's reads

$$
\mathrm{i} \varphi_{t}=\Lambda \varphi-\frac{1}{2} \varphi_{z z}+V \varphi
$$

with $\Lambda_{j k}=\frac{1}{2}\left(\frac{x_{k}}{L_{r}}\right)^{2} \delta_{j k}$. Consider also the corresponding eigenvalue problem with the electric field turned off,

$$
\Lambda \varphi-\frac{1}{2} \varphi_{z z}+W \varphi=\lambda \varphi
$$

We will use the first eigenfunction of (3) as initial condition. This is the BesselGalerkin approximation of $u_{0}$ in (1). The eigenfunctions will have two continuous derivatives and be smooth away from the origin. Define the jump at the origin as

$$
[u]=\lim _{z \searrow 0} u(z)-\lim _{z \nearrow 0} u(z)
$$


The eigenfunctions of (3) will have the jump

$$
\left[\boldsymbol{\varphi}_{z z z}\right]=2\left[W^{\prime}\right] \boldsymbol{\varphi}(0)
$$

in their third derivatives. There will be jumps in the higher order derivatives as well. The jump in the derivative of the potential is

$$
\left[W_{j k}^{\prime}\right]=\frac{4}{L_{r}^{2} J_{1}\left(x_{j}\right) J_{1}\left(x_{k}\right)}
$$

Since the time-dependent part of the potential is smooth, $\left[V^{\prime}\right] \equiv\left[W^{\prime}\right]$.

For the time discretisation we will use the Magnus midpoint rule, using the Arnoldi process for evaluating the matrix exponentials. The Magnus midpoint rule is described in the following theorem, which shows second order accuracy for the method. Denote by $W^{k, p}$ the Sobolev space with $k$ derivatives bounded in $L_{p}$-norm, and by $H^{k}=W^{k, 2}$ the corresponding Hilbert spaces.

Theorem 1. [7, Theorem 3.1] Consider the abstract Schrödinger equation

$$
\begin{aligned}
& u^{\prime}(t)=-\mathrm{i} H(t) u(t), \quad-\infty<t<\infty, \\
& u(0)=u_{0} \in H^{1},
\end{aligned}
$$

where $H(t)=-\frac{1}{2} \frac{\partial^{2}}{\partial z^{2}}+V(t)$ is self-adjoint in $L_{2}$. Discretise time as $t_{n}=n \Delta t$, and consider the Magnus midpoint rule,

$$
u_{n+1}=\mathrm{e}^{\Omega_{n}} u_{n},
$$

where

$$
\Omega_{n}=-\mathrm{i} \Delta t H\left(t_{n+\frac{1}{2}}\right) .
$$

If the bounds on the potential

$$
\left\|\frac{\mathrm{d}^{m}}{\mathrm{~d} t^{m}} V(t)\right\|_{L_{2}} \leq M_{m}, \quad m=0,1,2,
$$

and the commutator bound

$$
\|[H(\tau), H(\sigma)] v\| \leq K_{1} \Delta t\|v\|_{H^{1}} \quad \forall v \in H^{1},|\tau-\sigma| \leq \Delta t
$$

hold, then

$$
\left\|u\left(t_{n}\right)-u_{n}\right\| \leq C \Delta t^{2} t_{n} \max _{0 \leq t \leq t_{n}}\|u(t)\|_{H^{1}} .
$$

Here and in the rest of the paper, $C$ denotes a generic constant which may be different at each occasion, but stays independent of any relevant quantities such as step lengths. In order to fulfil the assumptions of Theorem 1 we need to require a few properties of the potential. We assume that at any time,

$$
V \in W^{1, \infty},\left.\quad V\right|_{z \geq 0},\left.V\right|_{z \leq 0} \in W^{2, \infty} .
$$

$V_{z}$ is then continuous except for a jump at $z=0$. We further assume

$$
\frac{\partial^{m}}{\partial t^{m}} V \in L_{2}, m=0,1,2, \quad \dot{V} \in W^{2, \infty}, \quad\left[V\left(t_{m}\right), V\left(t_{n}\right)\right]=0 .
$$


The eigenfunctions to (3) will reside in the linear space

$Y=\left\{u \in H^{\frac{7}{2}-\varepsilon}(\mathbb{R}):\left.u\right|_{\mathbb{R}_{+}} \in H^{\frac{9}{2}-\varepsilon}\left(\mathbb{R}_{+}\right),\left.u\right|_{\mathbb{R}_{-}} \in H^{\frac{9}{2}-\varepsilon}\left(\mathbb{R}_{-}\right),\left[u_{z z z}\right]=2\left[W^{\prime}\right] u(0)\right\}$

for any $\varepsilon>0$. The Hamiltonian can be seen as an operator $H(t): D(H) \rightarrow$ $H^{\frac{5}{2}-\varepsilon}$, where $D(H)=Y \subseteq H^{\frac{5}{2}-\varepsilon}$. We can then prove the following regularity result, which says that if the initial condition is in $Y$, the solution will stay in $Y$. This gives us knowledge of the jump in the lowest-order discontinuous derivative, making it possible to compensate for it. The proof builds on the theory of strongly continuous one-parameter unitary groups, which is reviewed in e.g. [18, Ch. 5].

Theorem 2. Assume that the initial condition $u_{0}$ in (4) is an element of $Y$. Then the Magnus midpoint rule approximation of the solution at time $t_{n}, u_{n}$, is also in $Y$ for any $n \in \mathbb{Z}$.

Proof. Since $H(t)$ is self-adjoint, $-\mathrm{i} H(\tau)$ at any fixed time $\tau$ is the infinitesimal generator of a strongly continuous one-parameter unitary group $\left\{S_{\tau}(t)\right\}_{t}$. Let $u_{0} \in D(H)$. By the properties $S_{\tau}(t+s)=S_{\tau}(t) S_{\tau}(s)=S_{\tau}(s) S_{\tau}(t), S_{\tau}(0)=I$ of a one-parameter group, and by the strong continuity of $\left\{S_{\tau}(t)\right\}_{t}$,

$$
\frac{\mathrm{d}}{\mathrm{d} t}\left(S_{\tau}(t) u_{0}\right)=\lim _{s \rightarrow 0} S_{\tau}(t) \frac{1}{s}\left(S_{\tau}(s)-I\right) u_{0}=-\mathrm{i} S_{\tau}(t) H(\tau) u_{0} .
$$

Likewise,

$$
\frac{\mathrm{d}}{\mathrm{d} t}\left(S_{\tau}(t) u_{0}\right)=\lim _{s \rightarrow 0} \frac{1}{s}\left(S_{\tau}(s)-I\right) S_{\tau}(t) u_{0}=-\mathrm{i} H(\tau) S_{\tau}(t) u_{0} .
$$

Therefore,

$$
H(\tau) S_{\tau}(t) u_{0}=S_{\tau}(t) H(\tau) u_{0} \quad \forall t, \tau \in \mathbb{R} .
$$

Since $H(\tau): D(H) \rightarrow H^{\frac{5}{2}-\varepsilon}$ and $S_{\tau}(t): H^{\frac{5}{2}-\varepsilon} \rightarrow H^{\frac{5}{2}-\varepsilon}$, the right-hand side is an element in $H^{\frac{5}{2}-\varepsilon}$. Since we have shown equality, the left-hand side must also be an element in $H^{\frac{5}{2}-\varepsilon}$. Therefore must $S_{\tau}(t) u_{0} \in D(H)$. We can identify $\mathrm{e}^{\Omega_{n}}=S_{t_{n+\frac{1}{2}}}(\Delta t)$. Hence, $u_{n} \in D(H) \Rightarrow u_{n+1} \in D(H)$, and the result follows by induction.

\section{Absorbing boundary conditions}

In order to make numerical simulation we need to truncate the domain also in the axial direction. We do so at $z= \pm \frac{L_{z}}{2}$, where we apply absorbing boundary conditions. The problem at hand is not really an outflow problem. The slope of the potential perpetually changes directions due to the electric field and there is therefore no point of no return, the wave function may always, regardless of axial coordinate, move towards the origin. However, we are mostly concerned with the recombination process in which the higher harmonics are generated, and the likelihood of an electron returning to a bound orbit decreases the farther away from the nucleus it has gone. If recombination has not occurred within one period of the electric field, the electron is likely to never return. Absorbing boundary conditions is therefore a feasible approach. We implement absorbing boundaries using the modal perfectly matched layer (PML) presented in [13]. 
Consider as an instructional model the TDSE

$$
\mathrm{i} u_{t}=-\frac{1}{2} u_{z z}+V u, \quad 0 \leq z \leq L,
$$

for which we will implement a PML at the $z=L$ boundary. Let $f(z)=$ $1+\mathrm{e}^{\mathrm{i} \gamma} \sigma(z)$, where $0<\gamma<\frac{\pi}{2}$, and the damping function $\sigma(z)$ is compactly supported in the layer $[L, L+d]$. A typical choice is a polynomial damping function, $\sigma(z)=\sigma_{0}\left(\frac{z-L}{d}\right)^{p} \theta(z-L)$, where $\theta(z)$ denotes the Heaviside step function. The corresponding PML equation reads

$$
\mathrm{i} v_{t}=-\frac{1}{2} \frac{1}{f} \frac{\partial^{2}}{\partial z^{2}}\left(\frac{1}{f} v\right)+V_{P M L} v+V v,
$$

with

$$
V_{P M L}=\frac{1}{2} \frac{3 f^{\prime 2}-2 f^{\prime \prime} f}{4 f^{4}}
$$

and the substitution of variables $v=\sqrt{f} u$. We will use such PMLs in both ends of the domain, in the sub-intervals $\left[-\frac{1}{2} L_{z},-\frac{1}{2} L_{z}+d\right]$ and $\left[\frac{1}{2} L_{z}-d, \frac{1}{2} L_{z}\right]$, with the parameters $p=4, \sigma_{0}=20$, and $\gamma=\frac{1}{4}$. $d$ will take different values in the examples considered. The PML efficiently dampens the wave function near the boundaries of the computational domain. We will shortly discretise the axial direction using the Fourier pseudospectral method. That introduces periodic boundary conditions, which breaks the assumptions (5) and (6) since the potential is discontinuous at the periodic boundary. But due to the PML, the wave function is effectively zero near the boundary. The discontinuity of the potential therefore has negligible implications in practice.

\section{Axial discretisation}

In this section we discuss the discretisation in the axial direction. We first show how application of the Fourier pseudospectral method yields second order convergence as the axial step is reduced. We then introduce a correction term, which is the main contribution of this paper, and prove fourth order accuracy for the corrected Fourier method.

Introduce the equidistant axial grid $z_{j}=h j, j=-\frac{N_{z}}{2}, \ldots, \frac{N_{z}}{2}-1$. The equation, semi-discretised with the Fourier pseudospectral method, reads

$$
\mathrm{i} \dot{\phi}=\Lambda \otimes I_{N_{r}} \phi-\frac{1}{2} I_{N_{r}} \otimes D \phi+I_{N_{r}} \otimes V \phi-z e(t) \phi .
$$

Here, $\otimes$ denotes the Kronecker product and $D$ is the Fourier second derivative differentiation matrix. In actual computations we will not explicitly multiply with the matrix $D$, but compute its action on $\phi_{k}$ using the fast Fourier transform.

Consider the TDSE (2), where $V(z, t)$ is a symmetric-matrix-valued function which fulfils the conditions (5) and (6). It is even in $z$ and has no continuous derivatives at the origin but is smooth elsewhere. For our convergence results we will make use of the well-established result for the spectral accuracy of Fourier interpolation. 
Theorem 3. [11, 14] If $u \in H^{s}, s>\frac{1}{2}$, and $v$ is its $N$-mode Fourier interpolant, then for any $0 \leq \sigma \leq s$,

$$
\|u-v\|_{H^{\sigma}} \leq C_{\sigma}\|u\|_{H^{s}} \frac{1}{N^{s-\sigma}} .
$$

In the following lemma we derive the truncation error of the Fourier pseudospectral approximation of the second derivative, when applied to a function in $Y$. By $\left.u\right|_{x_{j}}$ we denote the function $u(x, t)$ evaluated in the point $x_{j}$.

Lemma 1. If the periodic function $u:[-a, a] \rightarrow \mathbb{C}$ is smooth except for finite jumps at $x=0$ in its third and possibly higher order derivatives, the truncation error for the Fourier pseudospectral approximation of its second derivative at $x_{j}=j h, \tau_{j}=\left.u_{x x}\right|_{x_{j}}-D u_{j}, j=-\frac{N}{2}, \ldots, \frac{N}{2}-1$, is bounded by

$$
\left|\tau_{j}\right| \leq C \frac{h^{1-\varepsilon}}{1+j^{2}}+\mathcal{O}\left(h^{2}\right)
$$

where $\varepsilon>0$ can be arbitrarily small.

Proof. Let $v$ be the Fourier interpolant of $u$, and let $e=u-v . D u=v_{x x}$ and $u_{x x}-D u=e_{x x}$. By Sobolev's inequality, for any $k>\frac{1}{2}$,

$$
\left\|e_{x x}\right\|_{L^{\infty}} \leq C\left\|e_{x x}\right\|_{H^{k}} \leq C\|e\|_{H^{k+2}} .
$$

Since $u \in H^{\frac{7}{2}-\frac{\varepsilon}{2}}, \varepsilon>0$, by Theorem 3 ,

$$
\|e\|_{H^{2}} \leq C\|u\|_{H^{\frac{7}{2}-\frac{\varepsilon}{2}}} h^{\frac{7}{2}-\frac{\varepsilon}{2}-2}
$$

which gives us

$$
\left\|e_{x x}\right\|_{L^{\infty}} \leq C\|u\|_{H^{\frac{7}{2}-\frac{\varepsilon}{2}}} h^{\frac{3}{2}-\frac{\varepsilon}{2}-k}
$$

Since $e_{x x}$ is an interpolant of $\left\{\tau_{j}\right\}$, choosing $k=\frac{1}{2}+\frac{\varepsilon}{2}$,

$$
\left|\tau_{j}\right| \leq C h^{1-\varepsilon} \forall j
$$

with $C$ independent of $h$. Considering a formally infinite domain, the Fourier differentiation operator $D$ corresponds to the difference scheme

$$
D u_{j}=\frac{1}{h^{2}} \sum_{n=-\infty}^{\infty} \beta_{n} u_{j+n},
$$

with

$$
\beta_{n}=\left\{\begin{array}{cc}
\frac{2(-1)^{n+1}}{n^{2}}, & n \neq 0 \\
-\frac{\pi^{2}}{3}, & n=0
\end{array}\right.
$$

Using the periodicity we treat $u$ as a function defined on all of $\mathbb{R}$. We can make the decomposition $u=u^{(1)}+u^{(2)}$, where $u^{(2)} \in C^{3}$, and $u^{(1)}=0$ in $(0,2 a)$. Furthermore, we can require that $u^{(1)}$ does not change sign in $x>2 a$ or in 
$x<0$. Then for $0<j<\frac{N}{2}$,

$$
\begin{aligned}
\tau_{j} & =D u_{j}^{(1)}-\underbrace{\left.u_{x x}^{(1)}\right|_{x_{j}}}_{0}+\underbrace{D u_{j}^{(2)}-\left.u_{x x}^{(2)}\right|_{x_{j}}}_{\mathcal{O}\left(h^{2}\right)}, \\
\left|\tau_{j}\right| & \leq\left|\frac{1}{h^{2}} \sum_{n=-\infty}^{-j} \beta_{n} u_{j+n}+\frac{1}{h^{2}} \sum_{n=N-j}^{\infty} \beta_{n} u_{j+n}\right|+\mathcal{O}\left(h^{2}\right) \\
& \leq \frac{\left\|u^{(1)}\right\|_{L^{\infty}}}{h^{2}}\left(\left|\sum_{n=-\infty}^{-j} \beta_{n}\right|+\left|\sum_{n=N-j}^{\infty} \beta_{n}\right|\right)+\mathcal{O}\left(h^{2}\right) \\
& \leq \frac{\left\|u^{(1)}\right\|_{L^{\infty}}}{h^{2}}\left(\left|\beta_{-j}\right|+\left|\beta_{N-j}\right|\right)+\mathcal{O}\left(h^{2}\right) \\
& \leq \frac{C}{j^{2}}+\mathcal{O}\left(h^{2}\right) .
\end{aligned}
$$

We can make a similar decomposition for $-\frac{N}{2} \leq j<0$.

We will now prove the convergence rate for the standard Fourier method applied to the problem at hand. In the analysis we will drop the potential term. Since the potential is self-adjoint it vanishes in the energy estimate for the error equation. It is furthermore, in contrast to differentiation, a bounded operator, at high resolution the derivative will dominate the discretised Hamiltonian.

Theorem 4. Consider the TDSE (4) without potential. The initial condition, and therefore also the solution, resides in the space $Y$. Consider also its semidiscretisation

$$
\mathrm{i} \dot{v}=-\frac{1}{2} D v,
$$

where $v$ is a linear combination of Fourier modes. The error, defined as

$$
e_{j}(t)=u\left(z_{j}, t\right)-v_{j}(t)
$$

is bounded by

$$
\sqrt{\int_{0}^{T}\|\mathbf{e}(t)\|^{2} \mathrm{~d} t} \leq C_{T} h^{2-\varepsilon}
$$

for any $\varepsilon>0 . C_{T}$ depends on $T$ but is independent of $h$.

Proof. The truncation error at $z_{j}, \tau_{j}$, is defined through

$$
\left.\frac{1}{2} D u\right|_{z_{j}}=\left.\frac{1}{2} u_{z z}\right|_{z_{j}}+\tau_{j} .
$$

By Theorem 3, since $u \in H^{\frac{7}{2}-\varepsilon}$ for any $\varepsilon>0$, the truncation error $\|\boldsymbol{\tau}\| \sim$ $\mathcal{O}\left(h^{\frac{3}{2}-\varepsilon}\right)$. The error equation reads

$$
\mathrm{i} \dot{\mathbf{e}}=-\frac{1}{2} D \mathbf{e}+\boldsymbol{\tau} .
$$

Since $D$ is self-adjoint, the energy method yields the bound

$$
\|\mathbf{e}(\cdot, t)\| \leq C h^{\frac{3}{2}-\varepsilon} t,
$$


which is not sharp. In order to get a stronger bound for $\mathbf{e}$ we use the Laplace transform technique [6]. We Laplace transform the error equation (9) in time,

$$
\begin{aligned}
\text { i } s \hat{\mathbf{e}} & =H \hat{\mathbf{e}}+\hat{\boldsymbol{\tau}} \\
\hat{\mathbf{e}} & =(\mathrm{i} s I-H)^{-1} \hat{\boldsymbol{\tau}} .
\end{aligned}
$$

Let $A(s)=\mathrm{i} s I-H$, and split $s$ in its real and imaginary parts, $s=\eta+\mathrm{i} \xi$. Assume $\eta \geq \eta_{0}>0$. By Parseval's relation,

$$
\begin{aligned}
\int_{0}^{\infty} \mathrm{e}^{-2 \eta t}\|\mathbf{e}(t)\|^{2} \mathrm{~d} t & =\frac{1}{2 \pi} \int_{-\infty}^{\infty}\|\hat{\mathbf{e}}(\eta+\mathrm{i} \xi)\|^{2} \mathrm{~d} \xi \\
& =\frac{1}{2 \pi} \int_{-\infty}^{\infty}\left\|A^{-1}(\eta+\mathrm{i} \xi) \hat{\boldsymbol{\tau}}(\eta+\mathrm{i} \xi)\right\|^{2} \mathrm{~d} \xi
\end{aligned}
$$

$A$ can be diagonalised by the discrete Fourier transform matrix $F, A=F^{*} S F$, where $S$ is diagonal,

$$
S_{n n}=\mathrm{i} s-\frac{1}{2}\left(\frac{2 \pi}{L_{z}}\right)^{2} n^{2}, \quad n=-\frac{N_{z}}{2}, \ldots, \frac{N_{z}}{2}-1 .
$$

Since $F$ is unitary, $A^{-1}=F^{*} S^{-1} F$. As a consequence of $\boldsymbol{\tau}$ fulfilling the conditions of Lemma 1, there exists a constant $C>0$ independent of $h$ such that

$$
C\|F \hat{\boldsymbol{\tau}}\|_{\infty} \leq\|F \hat{\boldsymbol{\tau}}\| \leq \sqrt{L_{z}}\|F \hat{\boldsymbol{\tau}}\|_{\infty} .
$$

Let $\mathbb{1}$ denote the vector with all elements equal to 1 .

$$
\begin{aligned}
\int_{0}^{\infty} \mathrm{e}^{-2 \eta t}\|\mathbf{e}(t)\|^{2} \mathrm{~d} t & =\frac{1}{2 \pi} \int_{-\infty}^{\infty}\left\|A^{-1}(\eta+\mathrm{i} \xi) \hat{\boldsymbol{\tau}}(\eta+\mathrm{i} \xi)\right\|^{2} \mathrm{~d} \xi \\
& =\frac{1}{2 \pi} \int_{-\infty}^{\infty}\left\|F^{*} S^{-1} F \hat{\boldsymbol{\tau}}\right\|^{2} \mathrm{~d} \xi \\
& \leq \frac{1}{2 \pi} \int_{-\infty}^{\infty}\left\|S^{-1} \mathbb{1}\right\|^{2}\|F \hat{\boldsymbol{\tau}}\|_{\infty}^{2} \mathrm{~d} \xi \\
& \leq C \int_{-\infty}^{\infty}\left\|S^{-1} \mathbb{1}\right\|^{2}\|\hat{\boldsymbol{\tau}}\|^{2} \mathrm{~d} \xi \\
& \leq C h \int_{-\infty}^{\infty}\left(\sum_{n=-\frac{N_{z}}{2}}^{\frac{N_{z}}{2}-1}\left|\frac{1}{\mathrm{i} \eta-\xi-\frac{1}{2}\left(\frac{2 \pi}{L_{z}}\right)^{2} n^{2}}\right|^{2}\right)\|\hat{\boldsymbol{\tau}}\|^{2} \mathrm{~d} \xi
\end{aligned}
$$

The time-derivative of the truncation error can be bounded by $\|\dot{\boldsymbol{\tau}}(t)\| \leq C h^{\frac{3}{2}-\varepsilon} \mathrm{e}^{\alpha t}$. We can then bound its Laplace transform by

$$
\|\hat{\boldsymbol{\tau}}\| \leq h^{\frac{3}{2}-\varepsilon} \frac{C}{|s|}
$$

for $\operatorname{Re} s>\alpha$. The integral can then be bounded, independently of $N_{z}$, by

$$
\int_{-\infty}^{\infty}\left(\sum_{n=-\frac{N_{z}}{2}}^{\frac{N_{z}}{2}-1}\left|\frac{1}{i \eta-\xi-\frac{1}{2}\left(\frac{2 \pi}{L_{z}}\right)^{2} n^{2}}\right|^{2}\right)\|\hat{\boldsymbol{\tau}}\|^{2} \mathrm{~d} \xi \leq \frac{C h^{3-2 \varepsilon}}{\eta^{2}} .
$$


We then get the bound

$$
\int_{0}^{\infty} \mathrm{e}^{-2 \eta t}\|\mathbf{e}(t)\|^{2} \mathrm{~d} t \leq \frac{C h^{4-2 \varepsilon}}{\eta^{2}} .
$$

Truncating the time interval yields the result,

$$
\begin{gathered}
\int_{0}^{T} \mathrm{e}^{-2 \eta t}\|\mathbf{e}(t)\|^{2} \mathrm{~d} t \leq \frac{C h^{4-2 \varepsilon}}{\eta^{2}} \\
\int_{0}^{T}\|\mathbf{e}(t)\|^{2} \mathrm{~d} t \leq \mathrm{e}^{2 \eta T} \frac{C h^{4-2 \varepsilon}}{\eta^{2}} \\
\sqrt{\int_{0}^{T}\|\mathbf{e}(t)\|^{2} \mathrm{~d} t} \leq C_{T} h^{2-\varepsilon}
\end{gathered}
$$

We now make use of Theorem 2, which gives us knowledge about the jump in the third derivative of the solution. For any $\varphi \in Y$, the truncation error as defined in (8) is bounded by $\|\boldsymbol{\tau}\| \leq C h^{\frac{3}{2}-\varepsilon}$ by Theorem 3 since $Y \subset H^{\frac{7}{2}-\varepsilon}$. The dominant term in the truncation error is due to the jump in $\boldsymbol{\varphi}_{z z z}$. In the spirit of [8] we cancel this error term by subtracting the truncation error from numerical differentiation applied to a function with a similar jump. We define this function as

$$
\boldsymbol{\psi}=\left[W^{\prime}\right] w(z) \boldsymbol{\varphi}(0)
$$

We need to construct the function $w(z)$, which should have the jump $\left[w_{z z z}\right]=2$ at the origin, be infinitely smooth away from the origin, have known derivatives, and decay towards the boundaries so that it in practice is unaffected by the periodic wrapping. We choose

$$
w(z)=\left\{\begin{array}{cc}
\frac{1}{6} z^{3} \mathrm{e}^{-\alpha z^{p}}, & z>0 \\
-\frac{1}{6} z^{3} \mathrm{e}^{-\alpha z^{p}}, & z<0 .
\end{array}\right.
$$

Then $\boldsymbol{\varphi}-\boldsymbol{\psi} \in H^{\frac{9}{2}-\varepsilon}$. Exchanging $D \boldsymbol{\varphi}$ for $D(\boldsymbol{\varphi}-\boldsymbol{\psi})+\boldsymbol{\psi}_{z z}$ earns us one order of accuracy. By adding

$$
E=\left[W^{\prime}\right] \otimes\left(\frac{1}{2}\left(D \mathbf{w}-\mathbf{w}^{\prime \prime}\right) \mathbf{e}_{0}^{T}\right),
$$

where the vectors $\mathbf{w}$ and $\mathbf{w}^{\prime \prime}$ are $w$ and $w^{\prime \prime}$ put on the axial grid, to the Hamiltonian we cancel the effect of the jump in the third derivative, and the order of accuracy is increased. $\mathbf{e}_{0}$ is the vector with the value one in element 0 , corresponding to the origin, and zero elsewhere. Since $D$ is spectrally accurate, the added truncation error from differentiating the smooth parts of $\mathbf{w}$ is negligible.

$E$ is not Hermitian, and does therefore not vanish in the energy estimate. We get an energy estimate of the kind $\frac{\mathrm{d}}{\mathrm{d} t}\|u\| \leq \frac{1}{2}\left\|E^{*}-E\right\|\|u\|$. However, $\left\|E^{*}-E\right\|$ decays as $\mathcal{O}\left(h^{\frac{3}{2}-\varepsilon}\right)$ as the grid is refined, and we thereby maintain strict stability [6, Definition 5.1.2] if the PML, for which stability has not been established, is neglected. At sufficiently high resolution, which in our experience is not particularly high — well below the resolution required by accuracy requirements 
- the perturbation is small enough for the perturbed Hamiltonian to have distinct, real eigenvalues, as in the unperturbed case.

The truncation error for the corrected Fourier method satisfies the following bounds.

Lemma 2. If $u \in Y$, the truncation error $\boldsymbol{\tau}$ of the corrected Fourier pseudospectral method, defined as

$$
-\frac{1}{2} D\left(\left.u\right|_{z_{j}}-\left.\left.\left[W^{\prime}\right] w\right|_{z_{j}} u\right|_{0}\right)=-\left.\frac{1}{2} u_{z z}\right|_{z_{j}}+\left.\left.\frac{1}{2}\left[W^{\prime}\right] w^{\prime \prime}\right|_{z_{j}} u\right|_{0}-\tau_{j},
$$

satisfies the bound

$$
\|\boldsymbol{\tau}\| \leq C h^{\frac{5}{2}-\varepsilon}
$$

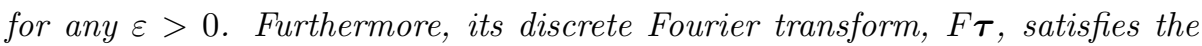
mode-wise bound

$$
|F \tau|_{n} \leq C h^{\frac{7}{2}-\varepsilon}|n|+\mathcal{O}\left(h^{\frac{7}{2}-\varepsilon}\right), \quad-\frac{N_{z}}{2} \leq n<\frac{N_{z}}{2} .
$$

Proof. Since $u \in Y, u-\left[W^{\prime}\right] w u(0, \cdot) \in H^{\frac{9}{2}-\varepsilon}$, and (11) follows directly by Theorem 3. Let

$$
f=-\frac{1}{2}\left(u-\left[W^{\prime}\right] w u(0, \cdot)\right),
$$

let $g$ be its Fourier interpolant, and assume for convenience that $L_{z}=1$. Denote the Fourier coefficients by $\tilde{f}_{n}=\int_{-\frac{1}{2}}^{\frac{1}{2}} f(z) \mathrm{e}^{-2 \pi \mathrm{i} n z} \mathrm{~d} z$. A component of the discrete Fourier transform,

$$
(F \mathbf{f})_{n}=\frac{1}{\sqrt{N_{z}}} \sum_{j=-\frac{N_{z}}{2}}^{\frac{N_{z}}{2}-1} f\left(z_{j}\right) \mathrm{e}^{-2 \pi \mathrm{i} j n / N_{z}}=\sqrt{N_{z}} \sum_{m=-\infty}^{\infty} \tilde{f}_{n+m N_{z}} .
$$

Then

$$
\begin{aligned}
\tau & =f_{z z}-g_{z z} \\
& =-\sum_{n=-\infty}^{\infty}(2 \pi n)^{2} \tilde{f}_{n} \mathrm{e}^{2 \pi \mathrm{i} n z}+\sum_{m=-\infty}^{\infty} \sum_{n=-\frac{N_{z}}{2}}^{\frac{N_{z}}{2}-1}(2 \pi n)^{2} \tilde{f}_{n+m N_{z}} \mathrm{e}^{2 \pi \mathrm{i} n z} \\
& =-\sum_{n=\frac{N_{z}}{2}}^{\infty}(2 \pi n)^{2} \tilde{f}_{n} \mathrm{e}^{2 \pi \mathrm{i} n z}-\sum_{n=-\infty}^{-\frac{N_{z}}{2}-1}(2 \pi n)^{2} \tilde{f}_{n} \mathrm{e}^{2 \pi \mathrm{i} n z}+\sum_{m \neq 0} \sum_{n=-\frac{N_{z}}{2}}^{\frac{N_{z}}{2}-1}(2 \pi n)^{2} \tilde{f}_{n+m N_{z}} \mathrm{e}^{2 \pi \mathrm{i} n z}, \\
(F \boldsymbol{\tau})_{n} & =-\sqrt{N_{z}} \sum_{m \neq 0} 4 \pi^{2}\left(n+m N_{z}\right)^{2} \tilde{f}_{n+m N_{z}}+\sqrt{N_{z}} \sum_{m \neq 0}(2 \pi n)^{2} \tilde{f}_{n+m N_{z}} \\
& =-4 \pi^{2} \sqrt{N_{z}} \sum_{m \neq 0}\left(m^{2} N_{z}^{2}+2 m n N_{z}\right) \tilde{f}_{n+m N_{z}} .
\end{aligned}
$$

Since $f \in H^{\frac{9}{2}-\varepsilon}$ its lowest order possible jump is in its fourth derivative, and

$$
\left|\tilde{f}_{n}\right| \leq \frac{C}{1+|n|^{5}} .
$$


The leading, $\mathcal{O}\left(h^{\frac{5}{2}-\varepsilon}\right)$, terms in the truncation error are due to a jump in the fourth derivative of $f$. Decomposing $f$ in its odd and even parts, $f=f^{\text {odd }}+$ $f^{\text {even }}$, only $f^{\text {odd }}$ can contribute to this jump. Therefore, $F \boldsymbol{\tau}=F \boldsymbol{\tau}^{\text {odd }}+\mathcal{O}\left(h^{\frac{7}{2}-\varepsilon}\right)$. Since $\left(F \boldsymbol{\tau}^{\text {odd }}\right)_{n}=-\left(F \boldsymbol{\tau}^{\text {odd }}\right)_{-n}$,

$$
\begin{aligned}
|F \boldsymbol{\tau}|_{n} & =\left|F \boldsymbol{\tau}^{\text {odd }}\right|_{n}+\mathcal{O}\left(h^{\frac{7}{2}-\varepsilon}\right) \\
& =\left|4 \pi^{2} \sqrt{N_{z}} \sum_{m \neq 0}\left(m^{2} N_{z}^{2}+2 m n N_{z}\right) \tilde{f}_{n+m N_{z}}^{\text {odd }}\right|+\mathcal{O}\left(h^{\frac{7}{2}-\varepsilon}\right) \\
& \leq C N_{z}^{\frac{5}{2}}\left|\sum_{m=1}^{\infty} m^{2}\left(\tilde{f}_{m N_{z}+n}^{\text {odd }}-\tilde{f}_{m N_{z}-n}^{\text {odd }}\right)\right|+C|n| N_{z}^{\frac{3}{2}}\left|\sum_{m=1}^{\infty} m\left(\tilde{f}_{m N_{z}+n}^{\text {odd }}+\tilde{f}_{m N_{z}-n}^{o d d}\right)\right|+\mathcal{O}\left(h^{\frac{7}{2}-\varepsilon}\right) .
\end{aligned}
$$

The second sum can be straight-forwardly bounded using the asymptotic decay of the Fourier coefficients. For the first sum we use the mean value theorem,

$$
\begin{aligned}
\left|\tilde{f}_{m N_{z}+n}^{o d d}-\tilde{f}_{m N_{z}-n}^{o d d}\right| & \leq 2|n| \max _{\nu \in\left[m N_{z}-n, m N_{z}+n\right]}\left|\frac{\mathrm{d}}{\mathrm{d} \nu} \tilde{f}_{\nu}^{o d d}\right| . \\
\frac{\mathrm{d}}{\mathrm{d} \nu} \tilde{f}_{\nu}^{o d d} & =\int_{-\frac{1}{2}}^{\frac{1}{2}} \tilde{f}_{\nu}^{o d d}(z) 2 \pi z \cos (2 \pi \nu z) \mathrm{d} z, \\
\left|\frac{\mathrm{d}}{\mathrm{d} \nu} \tilde{f}_{\nu}^{o d d}\right| & \leq C \frac{1}{1+\nu^{6}} . \\
\left|\tilde{f}_{m N_{z}+n}^{\text {odd }}-\tilde{f}_{m N_{z}-n}^{o d d}\right| & \leq C \frac{|n|}{1+\left(m N_{z}-n\right)^{6}} \\
& \leq C \frac{|n|}{1+\left(m N_{z}\right)^{6}} .
\end{aligned}
$$

We then arrive at the result,

$$
\begin{aligned}
|F \boldsymbol{\tau}|_{n} & \leq C|n| \sum_{m=1}^{\infty} \frac{m^{2} N_{z}^{\frac{5}{2}}}{1+\left(m N_{z}\right)^{6}}+C|n|\left|\sum_{m=1}^{\infty} \frac{m N_{z}^{\frac{3}{2}}}{1+\left(m N_{z}\right)^{5}}\right|+\mathcal{O}\left(h^{\frac{7}{2}-\varepsilon}\right) \\
& \leq C h^{\frac{7}{2}}|n|+\mathcal{O}\left(h^{\frac{7}{2}-\varepsilon}\right) .
\end{aligned}
$$

We can now prove the following error estimate for the corrected Fourier method.

Theorem 5. Consider the TDSE (4) without potential, and the semi-discretisation

$$
\mathrm{i} \dot{v}=-\frac{1}{2} D v+E v .
$$

The error, defined as

$$
e_{j}(t)=u\left(z_{j}, t\right)-v_{j}(t)
$$

is bounded by

$$
\sqrt{\int_{0}^{T}\|\mathbf{e}(t)\|^{2} \mathrm{~d} t} \leq C_{T} h^{4-\varepsilon}
$$

for any $\varepsilon>0 . C_{T}$ depends on $T$, but is independent of $h$. 
Proof. As before, we can derive the error equation

$$
\mathrm{i} \dot{\mathbf{e}}=-\frac{1}{2} D \mathbf{e}+E \mathbf{e}+\boldsymbol{\tau} .
$$

Lemma 2 gives the bound $\|\boldsymbol{\tau}\| \leq C h^{\frac{5}{2}-\frac{\varepsilon}{2}}$ on the truncation error. We assume that the resolution is enough for $H+E=-\frac{1}{2} D+E$ to have real eigenvalues. In order to bound $\mathbf{e}$ we Laplace transform the error equation,

$$
\begin{aligned}
\mathrm{i} s \hat{\mathbf{e}} & =(H+E) \hat{\mathbf{e}}+\hat{\boldsymbol{\tau}} \\
\hat{\mathbf{e}} & =(\mathrm{i} s I-H-E)^{-1} \hat{\boldsymbol{\tau}} .
\end{aligned}
$$

Let $B(s)=A(s)-E=\mathrm{i} s I-H-E$, and split $s=\eta+\mathrm{i} \xi$. Assume $\eta \geq \eta_{0}>0$. If $\eta$ is chosen big enough, such that $\left\|A^{-1} E\right\|<1$, we can apply the perturbation result [5, Theorem 2.3.4]. Writing $B^{-1}=A^{-1}+R$, the perturbation $R$ can be bounded by

$$
\|R\| \leq \frac{\|E\|\left\|A^{-1}\right\|^{2}}{1-\left\|A^{-1} E\right\|} .
$$

Since the non-zero column of $E$ is the truncation error from the Fourier second derivative of a $H^{\frac{7}{2}-\frac{\varepsilon}{2}}$-function, the bound $\|E\| \leq\|E\|_{F} \leq C h^{\frac{3}{2}-\frac{\varepsilon}{2}}$ holds. $\left\|A^{-1}\right\|$ can as before be bounded by a constant, and thereby $\|R\| \leq C h^{\frac{3}{2}-\frac{\varepsilon}{2}}$. By Parseval's relation,

$$
\begin{aligned}
\int_{0}^{\infty} \mathrm{e}^{-2 \eta t}\|\mathbf{e}(t)\|^{2} \mathrm{~d} t & =\frac{1}{2 \pi} \int_{-\infty}^{\infty}\|\hat{\mathbf{e}}(\eta+\mathrm{i} \xi)\|^{2} \mathrm{~d} \xi \\
& =\frac{1}{2 \pi} \int_{-\infty}^{\infty}\left\|B^{-1}(\eta+\mathrm{i} \xi) \hat{\boldsymbol{\tau}}(\eta+\mathrm{i} \xi)\right\|^{2} \mathrm{~d} \xi \\
& =\frac{1}{2 \pi} \int_{-\infty}^{\infty}\left\|A^{-1}(\eta+\mathrm{i} \xi) \hat{\boldsymbol{\tau}}(\eta+\mathrm{i} \xi)+R \hat{\boldsymbol{\tau}}(\eta+\mathrm{i} \xi)\right\|^{2} \mathrm{~d} \xi \\
& \leq \frac{1}{\pi} \int_{-\infty}^{\infty}\left\|A^{-1}(\eta+\mathrm{i} \xi) \hat{\boldsymbol{\tau}}(\eta+\mathrm{i} \xi)\right\|^{2} \mathrm{~d} \xi+\frac{1}{\pi} \int_{-\infty}^{\infty}\|R \hat{\boldsymbol{\tau}}(\eta+\mathrm{i} \xi)\|^{2} \mathrm{~d} \xi .
\end{aligned}
$$

Using (11), the bound on $\|R\|$, and that the truncation error grows in time with bounded rate, the integrand of the second integral can be bounded by

$$
\|R \hat{\tau}\|^{2} \leq\|R\|^{2}\|\hat{\boldsymbol{\tau}}\|^{2} \leq C h^{3-\varepsilon} h^{5-\varepsilon} \frac{1}{|s|^{2}}
$$

and

$$
\frac{1}{\pi} \int_{-\infty}^{\infty}\|R \hat{\boldsymbol{\tau}}(\eta+\mathrm{i} \xi)\|^{2} \mathrm{~d} \xi \leq C h^{8-2 \varepsilon} .
$$


We can derive a bound on the first integral using (12),

$$
\begin{aligned}
\frac{1}{\pi} \int_{-\infty}^{\infty}\left\|A^{-1} \hat{\boldsymbol{\tau}}\right\|^{2} \mathrm{~d} \xi & =\frac{1}{\pi} \int_{-\infty}^{\infty}\left\|F^{*} S^{-1} F \boldsymbol{\tau}\right\|^{2} \mathrm{~d} \xi \\
& =\frac{1}{\pi} \int_{-\infty}^{\infty}\left\|S^{-1} F \hat{\boldsymbol{\tau}}\right\|^{2} \mathrm{~d} \xi \\
& \leq C \int_{-\infty}^{\infty}\left(\left.h \sum_{n=-\frac{N_{z}}{2}}^{\frac{N_{z}}{2}-1} \frac{1}{\mathrm{i} \eta-\xi-\frac{1}{2}\left(\frac{2 \pi}{L_{z}}\right)^{2} n^{2}}\right|^{2} \frac{h^{7-2 \varepsilon}\left(n^{2}+1\right)}{|s|^{2}}\right) \mathrm{d} \xi \\
& \leq C h^{8-2 \varepsilon} \int_{-\infty}^{\infty}\left(\sum_{n=-\frac{N_{z}}{2}}^{\frac{N_{z}}{2}-1} \frac{1}{\eta^{2}+\left(\xi+\frac{1}{2}\left(\frac{2 \pi}{L_{z}}\right)^{2} n^{2}\right)^{2}} \frac{n^{2}+1}{\eta^{2}+\xi^{2}}\right) \mathrm{d} \xi
\end{aligned}
$$

The last integral can be bounded by a constant, independently of $N_{z}$. Put together,

$$
\int_{0}^{\infty} \mathrm{e}^{-2 \eta t}\|\mathbf{e}(t)\|^{2} \mathrm{~d} t \leq C h^{8-2 \varepsilon} .
$$

Truncating the time interval yields the result,

$$
\begin{gathered}
\int_{0}^{T} \mathrm{e}^{-2 \eta t}\|\mathbf{e}(t)\|^{2} \mathrm{~d} t \leq C h^{8-2 \varepsilon} \\
\int_{0}^{T}\|\mathbf{e}(t)\|^{2} \mathrm{~d} t \leq \mathrm{e}^{2 \eta T} C h^{8-2 \varepsilon} \\
\sqrt{\int_{0}^{T}\|\mathbf{e}(t)\|^{2} \mathrm{~d} t} \leq C_{T} h^{4-\varepsilon}
\end{gathered}
$$

where $C_{T}$ does not depend on $h$.

\section{$5 \quad$ Numerical examples}

We verify the derived convergence rates by computations on a model problem. We solve (7) with $L_{z}=40, L_{r}=5, N_{r}=3$, and PMLs of width $d=4$. As initial condition we use the first eigenstate of the discretised Hamiltonian. We solve up to the time $T=50$ using a monochromatic electric field, $e(t)=E_{0} \sin \left(\omega_{0} t\right)$. The field amplitude is $E_{0}=0.06$, and the frequency $\omega_{0}=0.1139$. In this parameter regime all characteristic features of the process are present. The convergence as $N_{z}$ is increased is shown in Figure 2 and Table 1. The reference solution was computed with $N_{z}=2048$. We experience second order convergence for the uncorrected Fourier method, and fourth order for the corrected, as expected. The time steps were chosen such that the time discretisation error is negligible, halving the time step does not change the numbers presented in the table.

We conclude with a simulation which illustrates the electron dynamics in the HHG process. We use a monochromatic electric with frequency $\omega_{0}=0.0570$, corresponding to the wavelength $800 \mathrm{~nm}$, and amplitude $E_{0}=0.06$, corresponding to an intensity of $1.26 \cdot 10^{14} \mathrm{Wcm}^{-2}$. The domain is truncated to the cylinder 


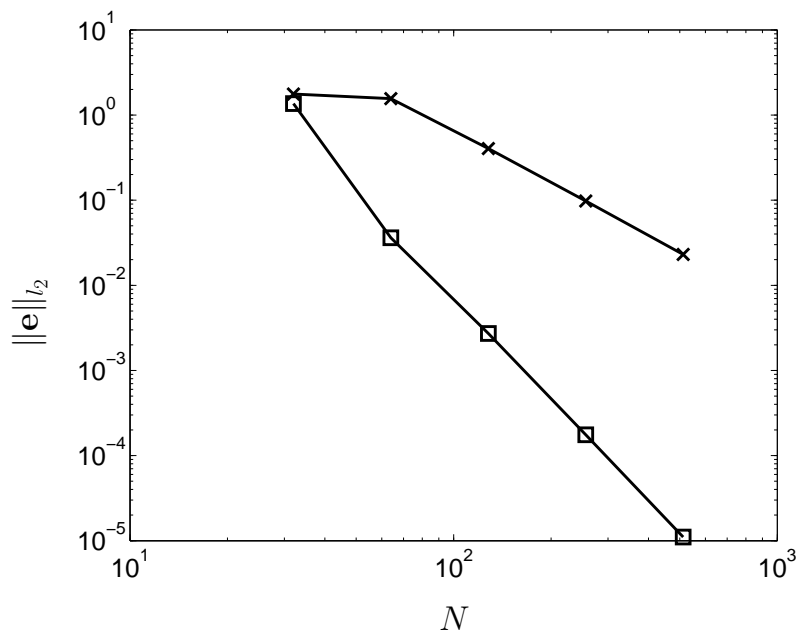

Figure 2: Decay of errors in $l_{2}$-norm as the axial grid is refined. The Fourier method converges at second order (crosses), the correction introduced in this paper accelerates convergence to fourth order (squares).

Table 1: Errors in $l_{2}$-norm for the Fourier method with $\left(\mathbf{e}_{4}\right)$ and without $\left(\mathbf{e}_{2}\right)$ correction.

\begin{tabular}{c|cc|cc}
$N_{z}$ & $\left\|\mathbf{e}_{2}\right\|$ & rate & $\left\|\mathbf{e}_{4}\right\|$ & rate \\
\hline 32 & 1.763 & - & 1.364 & - \\
64 & 1.560 & 0.18 & $3.617 \cdot 10^{-2}$ & 5.24 \\
128 & 0.4042 & 1.95 & $2.720 \cdot 10^{-3}$ & 3.73 \\
256 & $9.764 \cdot 10^{-2}$ & 2.05 & $1.756 \cdot 10^{-4}$ & 3.95 \\
512 & $2.308 \cdot 10^{-2}$ & 2.08 & $1.104 \cdot 10^{-5}$ & 3.99
\end{tabular}

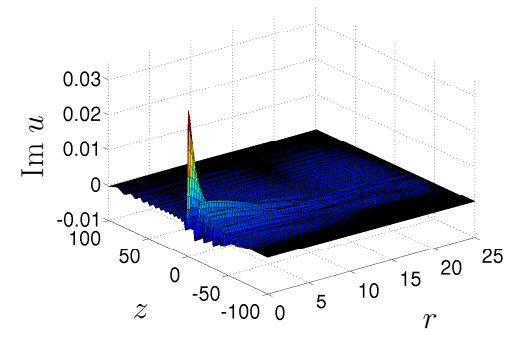

(a)

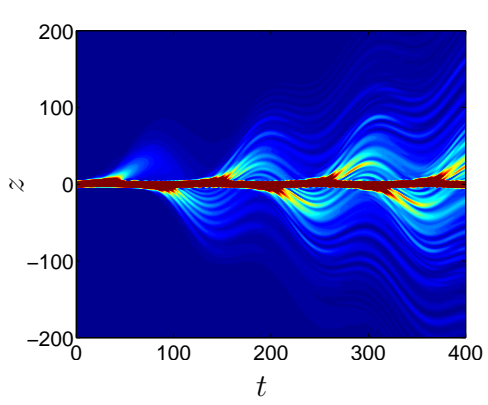

(b)

Figure 3: (a) The imaginary part of the wave function after one laser oscillation cycle, $t=110$. Most of the wave function is bound around the nucleus, but some have ionised and may generate higher harmonics. (b) The colours correspond to the probability density $\int|u|^{2} r \mathrm{~d} r$ of the electron having the axial coordinate $z$ at time $t$. The density at the core is out of the colour range in order to make the peripheral dynamics resolvable. 
$(z, r) \in[-210,210] \times[0,25]$, using 1024 Fourier modes in the axial direction and 64 Bessel functions in the radial direction. The PMLs have the width $d=4$. In Figure 3 (a) we show the imaginary part of the wave function at the time $t=110$, after one oscillation cycle of the laser. Most of the probability density remains in the bound states, the probability for tunnelling is comparably small. This will be the case for realistic field strengths, in experiment typically a few percent of the atoms will ionise. The wiggles at $z \gg 0$ are the parts of the wave function which tunnelled at the first peak of the electric field. Most of the then ionised wave function have at this point recombined with the nucleus or crossed to $z<0$. Most of what remains at $z \gg 0$ will not return, it represents permanent ionisation and will not contribute to the harmonic radiation. At $z \ll 0$ we see the parts of the wave function which tunnelled a half-cycle later, when the field peaked in the other direction. At the point in time pictured, the electric field is about to start accelerating this probability back towards the nucleus. In Figure 3 (b) we see the time-evolution of the probability density for the electron being at different $z$-coordinates. The probability density, given by the squared modulus of the wave function, is here integrated over $r$ in order to only picture the $z$-wise distribution. Note how the probability density blurs without expressed pattern after the first tunnelling point, but has distinct wiggles after subsequent tunnellings. The wiggles are the effect of interference between wave packets which tunnelled at different points in time, which cannot happen before the second tunnelling event. Note also how probability which has not recombined after one oscillation cycle continues to move away from the nucleus. This strengthens the claim that the interesting dynamics which is important for HHG happen near the nucleus, and that the use of absorbing boundary conditions is justified. It also emphasises that accuracy near the nucleus, which the correction presented in this paper improves, is important.

\section{Acknowledgements}

The author thanks Sverker Holmgren for helpful discussions, and Markus Kowalewski for providing access to his software for quantum dynamics computations.

\section{References}

[1] G. Doumy and L. F. DiMauro. Interrogating molecules. Science, 322:11941195, 2008.

[2] M. Drescher, M. Hentschel, R. Kienberger, M. Uiberacker, V. Yakovlev, A. Scrinzi, T. Westerwalbesloh, U. Kleineberg, U. Heinzmann, and F. Krausz. Time-resolved atomic inner-shell spectroscopy. Nature, 419:803$807,2002$.

[3] B. Fornberg. The pseudospectral method: Comparisons with finite differences for the elastic wave equation. Geophysics, 52:483-501, 1987.

[4] B. Fornberg. The pseudospectral method: Accurate representation of interfaces in elastic wave calculations. Geophysics, 53:625-637, 1988. 
[5] G. H. Golub and C. F. Van Loan. Matrix computations. Johns Hopkins University Press, Baltimore, MD, 3rd edition, 1996.

[6] B. Gustafsson, H.-O. Kreiss, and J. Oliger. Time Dependent Problems and Difference Methods. Wiley, New York, NY, 1995.

[7] M. Hochbruck and C. Lubich. On Magnus integrators for time-dependent Schrödinger equations. SIAM J. Numer. Anal., 41:945-963, 2003.

[8] J.-H. Jung. A note on the spectral collocation approximation of some differential equations with singular source terms. J. Sci. Comput., 39:4966, 2009.

[9] D. Kosloff and R. Kosloff. A Fourier method solution for the time dependent Schrödinger equation as a tool in molecular dynamics. J. Comput. Phys., 52:35-53, 1983.

[10] F. Krausz and M. Ivanov. Attosecond physics. Rev. Mod. Phys., 81:163$234,2009$.

[11] H.-O. Kreiss and J. Oliger. Stability of the Fourier method. SIAM J. Numer. Anal., 16:421-433, 1979.

[12] E. Luo and H.-O. Kreiss. Pseudospectral vs. finite difference methods for initial value problems with discontinuous coefficients. SIAM J. Sci. Comput., 20:148-163, 1998.

[13] A. Nissen and G. Kreiss. An optimized perfectly matched layer for the Schrödinger equation. Commun. Comput. Phys., 9:147-179, 2011.

[14] E. Tadmor. The exponential accuracy of Fourier and Chebyshev differencing methods. SIAM J. Numer. Anal., 23:1-10, 1986.

[15] E. Tadmor. Filters, mollifiers and the computation of the Gibbs phenomenon. Acta Numer., 16:305-378, 2007.

[16] G. N. Watson. A Treatise on the Theory of Bessel Functions. Cambridge University Press, Cambridge, 2nd edition, 1966.

[17] C. Winterfeldt, C. Spielmann, and G. Gerber. Colloquium: Optimal control of high-harmonic generation. Rev. Mod. Phys., 80:117-140, 2008.

[18] E. Zeidler. Applied Functional Analysis: Applications to Mathematical Physics. Springer-Verlag, New York, NY, 1995. 